\title{
Association of Single Nucleotide Polymorphisms of the IL-6, IL-10, and TNF- $\alpha$ Genes with Susceptibility to Gestational Diabetes Mellitus
}

\author{
Qing Wei, ${ }^{1}$ Xufeng Chen, ${ }^{2}$ and Heng Chen ${ }^{3}$
}

Objective: To investigate any associations between the single nucleotide polymorphisms (SNPs) at the interleukin-6 (IL-6) rs1800796, interleukin-10 (IL-10) rs1800896, and the tumor necrosis factor-alpha $(T N F-\alpha)$ rs1800629 loci with gestational diabetes mellitus (GDM) susceptibility.

Materials and Methods: A total of 242 GDM patients and 242 healthy controls were enrolled in this study. The genotypes of the $I L-6$ rs1800796, $I L-10$ rs1800896, and $T N F-\alpha$ rs1800629 loci were analyzed by Sanger sequencing. Interactions among these SNPs were analyzed through multifactor dimensionality reduction.

Results: Women with the $I L-6$ rs $1800796 \mathrm{G}$ allele had a higher GDM susceptibility risk than those with the C allele (odds ratio $[\mathrm{OR}]=1.44,95 \%$ confidence interval $[\mathrm{CI}]: 1.10-1.90, p=0.010$ ). Women with the $I L-10$ rs $1800896 \mathrm{C}$ allele had a higher risk of GDM susceptibility than those with the T allele (OR $=2.94,95 \% \mathrm{CI}$ : $1.87-4.63, p<0.001)$. Women with the $T N F-\alpha$ rs1800629 A allele had a higher risk of susceptibility to GDM than those with the $\mathrm{G}$ allele $(\mathrm{OR}=3.73,95 \% \mathrm{CI}: 2.25-6.18, p<0.001)$. The plasma levels of IL- 6 and TNF- $\alpha$ in GDM patients were significantly higher than those in the control group, and the levels of IL-10 were significantly lower than those in the control group $(p<0.001)$. Women with the IL-6 rs1800796 CG/GG genotypes had higher plasma IL-6 levels than those with the CC genotype $(p<0.05)$. Women with the IL-10 rs 1800896 TT genotype had higher IL-10 levels than those with the TC/CC genotypes $(p<0.05)$, and those with the TNF- $\alpha$ rs 1800629 GA/AA genotypes had higher TNF- $\alpha$ levels than those with the GG genotype $(p<0.05)$.

Conclusion: The results of this study show that the IL-6 rs1800796 G allele, the IL-10 rs $1800896 \mathrm{C}$ allele, and the TNF- $\alpha$ rs 1800629 A allele are significantly associated with an increased risk of susceptibility to GDM.

Keywords: gestational diabetes mellitus, IL-6, IL-10, TNF- $\alpha$, single nucleotide polymorphism

\section{Introduction}

G estational Diabetes mellitus (GDM) refers to a range of glucose metabolism abnormalities that occur or are first discovered during pregnancy. GDM is a special type of diabetes that can significantly increase the morbidity and mortality rates of pregnant women and their fetuses (Sacks, 2014; McIntyre et al., 2019; Zhang et al., 2019). The pathogenesis of GDM is not yet fully understood. The immunologic/inflammatory pathogenesis model is a prevalent topic. It is generally believed that insulin resistance and decreased insulin sensitivity, which can be caused by a myriad of different reasons, are the main causes of GDM (Yang et al., 2013; Tang et al., 2019; Feng et al., 2020).

Recent studies have shown that interleukin-6 (IL-6) and tumor necrosis factor-alpha $(\mathrm{TNF}-\alpha)$, as proinflammatory factors, play important roles in development of insulin resistance and GDM (Nergiz et al., 2014; Lacroix et al., 2019; Rancourt et al., 2020). Interleukin 10 (IL-10) is an important anti-inflammatory cytokine that can be synthesized and secreted by a variety of cells; it can inhibit production of inflammatory cytokines by a variety of inflammatory cells. IL-10 not only plays an important regulatory role in the body's immune response but it also plays a wider role in many tissues and organs, including its participation in cell activation, proliferation, and differentiation (Laffer et al., 2019; Zhang and Kuchroo, 2019). Studies have shown that plasma IL-10 levels in GDM patients are significantly lower than in healthy controls and that low levels of IL-10 and excess insulin resistance in GDM patients are negatively correlated, suggesting that low levels of IL-10 may increase insulin resistance in GDM patients (Charles et al., 2011; Kowalski et al., 2011; Yang et al., 2018).

\footnotetext{
${ }^{1}$ Department of Women's Health, Hangzhou Fuyang Women's and Children's Hospital, Hangzhou, China.

${ }^{2}$ Department of Obstetrics and Gynecology, The Second Affiliated Hospital of Zhejiang Chinese Medical University, Hangzhou, China.

${ }^{3}$ Department of Clinical Laboratory, The Second Affiliated Hospital of Zhejiang Chinese Medical University, Hangzhou, China.
} 
The $I L-6$ gene is located on the human chromosome 7p1521 ; it has a total length of $5 \mathrm{~kb}$ and consists of five exons and four introns. The gene has three transcription initiation sites (ATG) and three TATA sequences (TATA box). The noncoding region at the $5^{\prime}$ end is $\sim 300$ bp upstream of the start site and contains most of the regulatory sequences for the IL-6 gene expression, with its expression being mainly regulated at the transcriptional level. The human $I L-6$ gene promoter region contains several polymorphisms that can affect its transcription $(-597 \mathrm{G} / \mathrm{A},-572 \mathrm{G} / \mathrm{C}$, and $-174 \mathrm{G} / \mathrm{C}$, etc.). The 180 to 123 -bp region upstream of the IL-6 gene promoter is a key region for IL-6 gene transcription regulation (Ma et al., 2011; Chang et al., 2015). Revilla et al. (2002) found that the existence of IL-6 gene polymorphisms causes differing susceptibilities to multiple diseases among individuals. The presence of a G/C single nucleotide polymorphism (SNP) at the 174 th base upstream of the $I L-6$ gene promoter can affect its transcription, and since the concentration of IL-6 varies with the efficiency of its gene transcription, this allele affects the occurrence and development of numerous diseases.

The $I L-10$ gene is located at q32 of human chromosome 1 and its expressed IL-10 protein is a multifunctional, cellular immunosuppressive factor produced by Th2 cells; it is involved in inflammatory and immune responses and is currently recognized as an inflammatory and immune suppressive factor (Jin et al., 2019; Wang et al., 2019). There are multiple SNP sites in the $I L-10$ gene, among which are $1082 \mathrm{G} / \mathrm{A}, 819 \mathrm{C} / \mathrm{T}$, and $592 \mathrm{C} / \mathrm{A}$ that are located within the promoter region and potentially could affect gene transcription and expression levels (Jin et al., 2019; Kalani et al., 2019; Shahriyari et al., 2019).

The $T N F$ genes are located in a 7-kb DNA sequence within the short arm of chromosome 6 inside the MHC class III gene region. They consist of TNFA and TNFB, encoding TNF- $\alpha$ and TNF- $\beta$, respectively, each containing four exons and three introns. There are many SNPs in the $T N F-\alpha$ promoter, including $-1031 \mathrm{~T} / \mathrm{G},-863 \mathrm{C} / \mathrm{A},-851 \mathrm{C} / \mathrm{T},-419 \mathrm{G} / \mathrm{C},-376 \mathrm{G} / \mathrm{A},-308$ G/A, and -238 G/A. Among them, rs1800629 (-308 G/A) is known to be related to the TNF- $\alpha$ transcriptional level as transcriptional factors bind more strongly to the A allele, resulting in higher plasma TNF- $\alpha$ levels - twofold higher than that of subjects with the G allele (Warzocha et al., 1998).

In this study, we investigated the correlation between IL-6 rs1800796, IL-10 rs1800896, and TNF- $\alpha$ rs1800629 SNPs and their roles in regard to susceptibility to GDM.

\section{Materials and Methods}

\section{Subjects}

A total of 242 patients who were diagnosed with GDM after routine prenatal examination in our obstetrics and gynecology clinic from May 2017 to October 2019 were enrolled. The reference standard for a diagnosis of GDM is that of the International Association of the Diabetes and Pregnancy Study Groups criteria (International Association of Diabetes and Pregnancy Study Groups Consensus Panel 2010). According to the age and body mass index (BMI) matching of GDM patients, 242 healthy controls were selected. Those with chronic hypertension, diabetes, rheumatoid arthritis and other inflammatory diseases, cancer, and chronic renal insufficiency were excluded. Our research protocol was approved by the Medical Ethics Committee of Hangzhou Fufang Women's and Children's Hospital, and informed consent was obtained from all subjects.

\section{Obtaining clinical data and blood biochemical data from subjects}

The age, gestational age, BMI before pregnancy, BMI at birth, newborn body mass, and APGAR data were collected from patients' medical records, and the plasma glycosylated hemoglobin A1c (HbA1c) and glucose levels were measured at 0,1 , and $2 \mathrm{~h}$ during glucose stimulation.

\section{Genotypic analyses}

We used the QIAamp DNA Blood Mini Kit (Qiagen, Valencia, CA) to extract genomic DNA from the peripheral blood of the subjects. Sanger sequencing was used to analyze the genotypes of the IL-6 rs1800796, IL-10 rs1800896, and TNF- $\alpha$ rs 180062 loci. Genomic DNA samples from $20 \%$ of the subjects were randomly selected for repeated sequencing. The results of the sequencing runs were entirely consistent.

\section{Enzyme-linked immunosorbent assay}

We collected $3 \mathrm{~mL}$ of peripheral blood from the subjects, let it stand at room temperature for $1-2 \mathrm{~h}$, centrifuged it at $3000 \mathrm{r} / \mathrm{min}$ for $5 \mathrm{~min}$, removed the plasma, and stored it at $-80^{\circ} \mathrm{C}$. In accordance with the manufacturer's instructions, the enzyme-linked immunosorbent assay (ELISA) was used

Table 1. Comparison of Clinical Features and Biochemical Indicators of GDM Patients and CONTROL GRoups

\begin{tabular}{|c|c|c|c|}
\hline Clinical features & $G D M(\mathrm{n}=242)$ & Control $(\mathrm{n}=242)$ & $\mathrm{p}$ \\
\hline Age (years, mean $\pm S D)$ & $29.51 \pm 5.07$ & $29.74 \pm 4.90$ & 0.61 \\
\hline Gestational weeks $($ mean \pm SD) & $38.26 \pm 1.48$ & $38.16 \pm 0.82$ & 0.36 \\
\hline BMI before pregnancy $\left(\mathrm{kg} / \mathrm{m}^{2}\right.$, mean $\left.\pm \mathrm{SD}\right)$ & $23.86 \pm 2.06$ & $23.80 \pm 2.07$ & 0.75 \\
\hline BMI at birth $\left(\mathrm{kg} / \mathrm{m}^{2}\right.$, mean $\left.\pm \mathrm{SD}\right)$ & $29.94 \pm 2.23$ & $29.67 \pm 3.08$ & 0.27 \\
\hline Newborn body mass $(g$, mean \pm SD $)$ & $3321.11 \pm 550.74$ & $3292.95 \pm 432.67$ & 0.53 \\
\hline APGAR $($ mean \pm SD) & $9.81 \pm 0.51$ & $9.78 \pm 0.43$ & 0.49 \\
\hline $\mathrm{HbA} 1 \mathrm{c}(\%$, mean $\pm \mathrm{SD})$ & $5.54 \pm 1.13$ & $4.29 \pm 0.96$ & $<0.001$ \\
\hline Zero-h plasma glucose (mM) & $4.86 \pm 0.69$ & $4.32 \pm 1.19$ & $<0.001$ \\
\hline One-h plasma glucose (mM) & $10.16 \pm 1.82$ & $7.31 \pm 1.55$ & $<0.001$ \\
\hline Two-h plasma glucose (mM) & $8.88 \pm 1.33$ & $6.55 \pm 1.28$ & $<0.001$ \\
\hline
\end{tabular}

APGAR, activity, pulse, grimace, appearance, and respiration; BMI, body mass index; GDM, gestational diabetes mellitus; HbA1c, hemoglobin A1c; SD, standard deviation. 


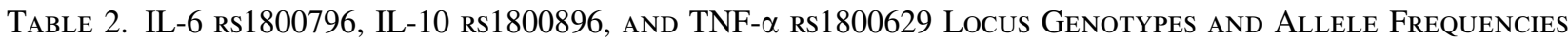

\begin{tabular}{|c|c|c|c|c|c|}
\hline & $G D M(\mathrm{n}=242)$ & Control $(\mathrm{n}=242)$ & $\begin{array}{c}H W E \\
\mathrm{p}\end{array}$ & $O R(95 \% C I)^{\mathrm{a}}$ & $\mathrm{p}$ \\
\hline \multicolumn{6}{|l|}{ IL-6 rs1800796 } \\
\hline $\mathrm{CC}$ & $107(44.21 \%)$ & $133(54.96 \%)$ & 0.06 & 1.00 & \\
\hline $\mathrm{CG}$ & $99(40.91 \%)$ & $85(35.12 \%)$ & & $1.45(0.98-2.13)$ & 0.074 \\
\hline GG & $36(14.88 \%)$ & $24(9.92 \%)$ & & $1.86(1.05-3.32)$ & 0.046 \\
\hline $\mathrm{CG}+\mathrm{GG}$ vs. $\mathrm{CC}$ & & & & $1.54(1.08-2.20)$ & 0.023 \\
\hline $\mathrm{GG}$ vs. $\mathrm{CG}+\mathrm{CC}$ & & & & $1.59(0.91-2.75)$ & 0.129 \\
\hline $\mathrm{C}$ & $313(64.67 \%)$ & $351(72.52 \%)$ & & 1.00 & \\
\hline $\mathrm{G}$ & $171(35.33 \%)$ & $133(27.48 \%)$ & & $1.44(1.10-1.90)$ & 0.010 \\
\hline \multicolumn{6}{|l|}{ IL-10 rs1800896 } \\
\hline TT & $184(76.03 \%)$ & $216(89.26 \%)$ & 0.16 & 1.00 & \\
\hline $\mathrm{TC}$ & $42(17.36 \%)$ & $24(9.92 \%)$ & & $2.05(1.20-3.52)$ & 0.012 \\
\hline $\mathrm{CC}$ & $16(6.61 \%)$ & $2(0.83 \%)$ & & $1.93(1.37-2.15)$ & 0.001 \\
\hline $\mathrm{TC}+\mathrm{CC}$ vs. TT & & & & $2.62(1.58-4.33)$ & $<0.001$ \\
\hline $\mathrm{CC}$ vs. $\mathrm{TC}+\mathrm{TT}$ & & & & $1.83(1.30-2.04)$ & 0.002 \\
\hline $\mathrm{T}$ & $410(84.71 \%)$ & $456(94.21 \%)$ & & 1.00 & \\
\hline $\mathrm{C}$ & $74(15.29 \%)$ & $28(5.79 \%)$ & & $2.94(1.87-4.63)$ & $<0.001$ \\
\hline \multicolumn{6}{|l|}{ TNF- $\alpha$ rs1800629 } \\
\hline GG & $191(78.93 \%)$ & $222(91.74 \%)$ & 0.40 & 1.00 & \\
\hline GA & $32(13.22 \%)$ & $19(7.85 \%)$ & & $1.96(1.08-3.57)$ & 0.038 \\
\hline AA & $19(7.85 \%)$ & $1(0.41 \%)$ & & $2.05(1.56-2.17)$ & $<0.001$ \\
\hline $\mathrm{GA}+\mathrm{AA}$ vs. GG & & & & $1.55(1.26-1.82)$ & $<0.001$ \\
\hline $\mathrm{AA}$ vs. GA+GG & & & & $1.98(1.50-2.08)$ & $<0.001$ \\
\hline $\mathrm{G}$ & $414(85.54 \%)$ & $463(95.66 \%)$ & & 1.00 & \\
\hline A & $70(14.46 \%)$ & $21(4.34 \%)$ & & $3.73(2.25-6.18)$ & $<0.001$ \\
\hline
\end{tabular}

${ }^{\mathrm{a}}$ Adjusted for age, gestational age, BMI before pregnancy, and BMI at birth.

$\mathrm{CI}$, confidence interval; HWE, Hardy-Weinberg equilibrium; IL, interleukin; OR, odds ratio; TNF- $\alpha$, tumor necrosis factor-alpha.

to detect levels of IL-6 (ab178013; Abcam, UK), IL-10 (ab133575; Abcam), and TNF- $\alpha$ (ab1793; Abcam) in plasma.

\section{Statistical analyses}

In this study, SPSS 20.0 (SPSS, Chicago, IL) was used for statistical analyses. Continuous variables were evaluated by the t-test and expressed as the mean \pm standard deviation. $n(\%)$ represents a categorical variable. Hardy-Weinberg equilibrium (HWE) was evaluated using the $\chi^{2}$ test for the statistical analysis. Odds ratios (ORs) and 95\% confidence intervals (CIs) were calculated to analyze the correlation between the genotypic and allelic frequencies of IL-6 rs1800796, IL-10 rs1800896, and TNF- $\alpha$ rs1800629 and GDM susceptibility and adjusted for the age, gestational week, BMI before pregnancy, and BMI at birth. In a multicomparison analysis, the Bonferroni correction was used to correct the $p$-value, and $p<0.0125(0.05 / 4)$ was considered to be significantly different. Multifactor dimensionality reduction (MDR) was used to analyze the IL-6 rs1800796, IL-10 rs 1800896 , and TNF- $\alpha$ rs 1800629 site interactions. The receiver operating curve (ROC) analysis was used to evaluate the diagnostic value of plasma for IL-6, IL-10, and TNF- $\alpha$ in GDM. All tests were two-tailed, and $p<0.05$ indicated a statistically significant difference.

\section{Results}

\section{Subject clinical characteristics}

The clinical signs and symptoms as well as biochemical indicators of the GDM patients and control group are shown in Table 1. There were no significant differences between the GDM patients and controls for age of patients, gestational weeks, BMI before pregnancy, BMI at birth, newborn body mass, and the activity, pulse, grimace, appearance, and respiration (APGAR) score (all $p$-values $<0.05$ ). The levels of plasma HbA1c and glucose were significantly lower among the control group compared with the GDM patients $(p>0.05)$.

\section{IL-6 rs1800796, IL-10 rs1800896, and TNF- $\alpha$ rs1800629 and GDM susceptibility}

The genotypic frequencies of the IL-6 rs1800796, IL-10 rs1800896, and TNF- $\alpha$ rs1800629 loci in the control group of

TABLE 3. GMDR ANALysis OF IL-6 Rs1800796, IL-10 Rs1800896, AND TNF- $\alpha$ Rs1800629 INTERACTIONS

\begin{tabular}{lcccccc}
\hline & Accuracy & Sensitivity & Specificity & Precision & OR $(95 \%$ CI) & $\mathrm{p}$ \\
\hline rs1800896 & $56.61 \%$ & $23.97 \%$ & $89.26 \%$ & $69.05 \%$ & $2.62(1.58-4.33)$ & $<0.001$ \\
rs1800896, rs1800629 & $61.16 \%$ & $40.08 \%$ & $82.23 \%$ & $69.29 \%$ & $3.10(2.04-4.70)$ & $<0.001$ \\
rs1800796, rs1800896, rs1800629 & $61.36 \%$ & $44.21 \%$ & $78.51 \%$ & $67.30 \%$ & $2.90(1.95-4.31)$ & $<0.001$
\end{tabular}

GMDR, generalized multifactor dimensionality reduction. 
A

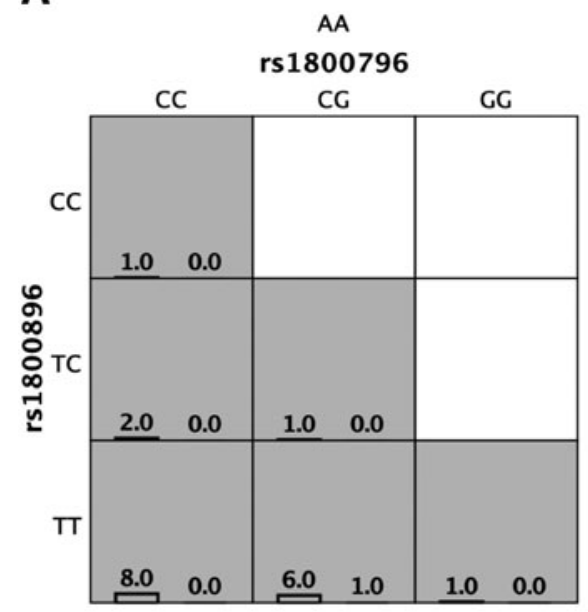

B

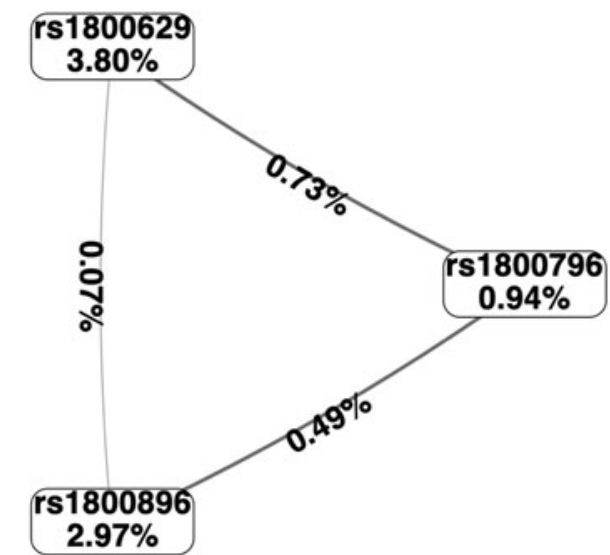

rs 1800629

GA

rs1800796

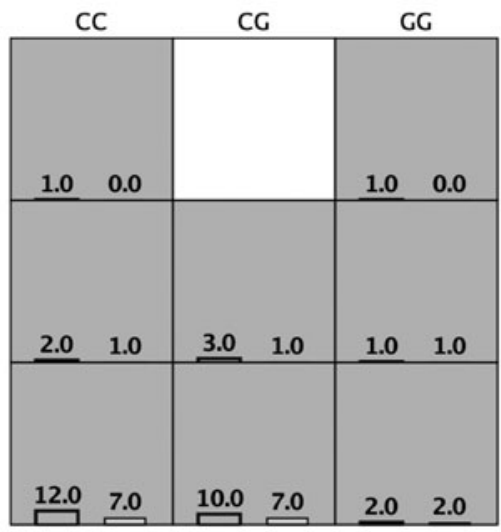

C

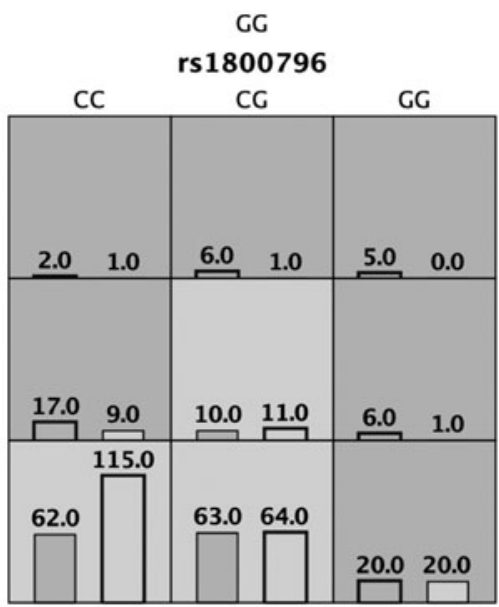

rs1800896

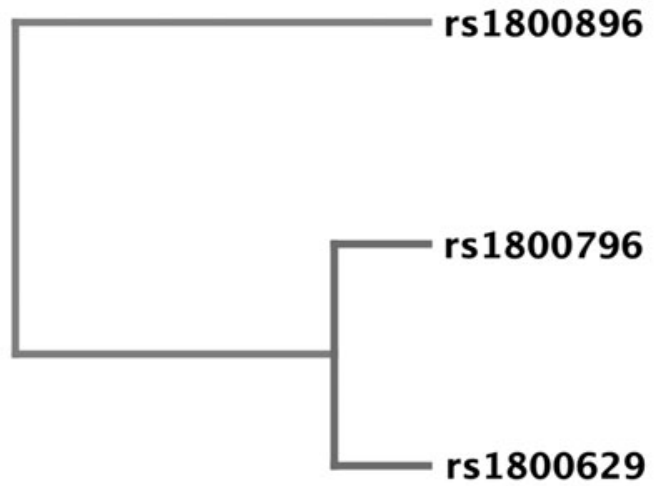

FIG. 1. MDR analysis of IL-6 rs1800796, IL-10 rs1800896, and TNF- $\alpha$ rs1800629 interactions. (A) Graphical model. In each box, the left bar represents the number of GDM patients and the right bar represents the number of subjects in the control group. (B) Circle graph. The bar boxes represent the SNP loci and the percentage represents the effect of the SNP loci on GDM susceptibility. The larger the data, the greater the impact of the SNP loci on GDM susceptibility. The data on the line represent the strength of the interactions between SNPs, and the larger the data represented, the stronger the interactions between SNPs. (C) Dendogram. The closer the SNP site, the stronger the interaction. GDM, gestational diabetes mellitus; HbA1c, hemoglobin A1c; IL, interleukin; MDR, multifactor dimensionality reduction; SNP, single nucleotide polymorphism; TNF- $\alpha$, tumor necrosis factor-alpha.

the study were in accordance with HWE $(p>0.05)$. The genotypic and allelic frequency distributions of IL-6 rs1800796, IL-10 rs1800896, and TNF- $\alpha$ rs1800629 are shown in Table 2. The IL-6 rs1800796 GG genotype under the dominant model was associated with an increased sus- ceptibility to $\mathrm{GDM}(\mathrm{OR}=1.86,95 \% \mathrm{CI}: 1.05-3.32, p=0.046$; $\mathrm{OR}=1.54,95 \%$ CI: $1.08-2.20, p=0.023)$. Persons with the $\mathrm{G}$ allele have a higher risk of susceptibility to GDM than those with the $\mathrm{C}$ allele $(\mathrm{OR}=1.44,95 \% \mathrm{CI}: 1.10-1.90, p=0.010)$. At the IL-10 rs1800896 loci, the TC and CC genotypes under

Table 4. Comparison of Clinical Characteristics of IL-6 rs1800796 Loci with Different Genotypes

\begin{tabular}{|c|c|c|c|c|c|c|}
\hline \multirow[b]{2}{*}{ Age (years) } & \multicolumn{2}{|c|}{$G D M$} & \multirow{2}{*}{$\frac{p}{0.30}$} & \multicolumn{2}{|c|}{ Control } & \multirow{2}{*}{$\begin{array}{c}\mathrm{p} \\
0.99\end{array}$} \\
\hline & $29.89 \pm 5.12$ & $29.21 \pm 5.03$ & & $29.73 \pm 5.42$ & $29.74 \pm 4.20$ & \\
\hline Gestational weeks & $38.26 \pm 1.30$ & $38.32 \pm 1.57$ & 0.75 & $38.21 \pm 0.76$ & $38.10 \pm 0.88$ & 0.30 \\
\hline BMI before pregnancy $\left(\mathrm{kg} / \mathrm{m}^{2}\right)$ & $23.95 \pm 2.35$ & $23.79 \pm 1.79$ & 0.56 & $23.79 \pm 2.12$ & $23.81 \pm 2.01$ & 0.94 \\
\hline BMI at birth $\left(\mathrm{kg} / \mathrm{m}^{2}\right)$ & $30.15 \pm 2.21$ & $29.76 \pm 2.23$ & 0.18 & $29.88 \pm 3.12$ & $29.41 \pm 3.02$ & 0.24 \\
\hline Newborn body mass (g) & $3269.30 \pm 518.09$ & $3362.17 \pm 573.89$ & 0.19 & $3295.50 \pm 429.05$ & $3289.83 \pm 439.01$ & 0.92 \\
\hline APGAR & $9.77 \pm 0.56$ & $9.82 \pm 0.50$ & 0.47 & $9.75 \pm 0.47$ & $9.82 \pm 0.39$ & 0.21 \\
\hline HbA1c $(\%)$ & $5.63 \pm 1.15$ & $5.47 \pm 1.12$ & 0.28 & $4.20 \pm 1.04$ & $4.39 \pm 0.86$ & 0.12 \\
\hline Zero-h plasma glucose (mM) & $4.81 \pm 0.66$ & $4.89 \pm 0.72$ & 0.37 & $4.25 \pm 1.25$ & $4.40 \pm 1.12$ & 0.33 \\
\hline One-h plasma glucose $(\mathrm{mM})$ & $10.09 \pm 1.89$ & $10.21 \pm 1.77$ & 0.61 & $7.37 \pm 1.57$ & $7.23 \pm 1.53$ & 0.49 \\
\hline Two-h plasma glucose (mM) & $8.92 \pm 1.39$ & $8.84 \pm 1.28$ & 0.64 & $6.58 \pm 1.26$ & $6.51 \pm 1.31$ & 0.67 \\
\hline
\end{tabular}


Table 5. Comparison of Clinical Characteristics of IL-10 rs1800896 Loci with Different Genotypes

\begin{tabular}{|c|c|c|c|c|c|c|}
\hline & \multicolumn{2}{|c|}{$G D M$} & \multirow[b]{2}{*}{$\mathrm{p}$} & \multicolumn{2}{|c|}{ Control } & \multirow[b]{2}{*}{$\mathrm{p}$} \\
\hline & $T T(\mathrm{n}=184)$ & $T C / C C(\mathrm{n}=58)$ & & $T T(\mathrm{n}=216)$ & $T C / C C(\mathrm{n}=26)$ & \\
\hline Age (years) & $29.44 \pm 5.07$ & $29.72 \pm 5.14$ & 0.72 & $29.79 \pm 4.92$ & $29.31 \pm 4.79$ & 0.64 \\
\hline Gestational weeks & $38.26 \pm 1.47$ & $38.28 \pm 1.54$ & 0.93 & $38.16 \pm 0.82$ & $38.15 \pm 0.78$ & 0.95 \\
\hline BMI before pregnancy $\left(\mathrm{kg} / \mathrm{m}^{2}\right)$ & $23.77 \pm 2.21$ & $24.14 \pm 2.36$ & 0.28 & $23.86 \pm 2.07$ & $23.34 \pm 1.99$ & 0.23 \\
\hline BMI at birth $\left(\mathrm{kg} / \mathrm{m}^{2}\right)$ & $29.91 \pm 2.21$ & $30.02 \pm 2.31$ & 0.74 & $29.72 \pm 3.11$ & $29.24 \pm 2.75$ & 0.45 \\
\hline Newborn body mass (g) & $3324.99 \pm 552.10$ & $3308.78 \pm 551.03$ & 0.85 & $3269.41 \pm 423.17$ & $3388.50 \pm 469.11$ & 0.21 \\
\hline APGAR & $9.80 \pm 0.52$ & $9.78 \pm 0.56$ & 0.80 & $9.78 \pm 0.44$ & $9.81 \pm 0.40$ & 0.74 \\
\hline $\mathrm{HbA1c}(\%)$ & $5.58 \pm 1.12$ & $5.41 \pm 1.17$ & 0.32 & $4.28 \pm 0.99$ & $4.35 \pm 0.75$ & 0.73 \\
\hline Zero-h plasma glucose (mM) & $4.89 \pm 0.71$ & $4.76 \pm 0.63$ & 0.21 & $4.39 \pm 1.14$ & $3.93 \pm 1.43$ & 0.06 \\
\hline One-h plasma glucose $(\mathrm{mM})$ & $10.15 \pm 1.81$ & $10.21 \pm 1.86$ & 0.83 & $7.29 \pm 1.55$ & $7.42 \pm 1.58$ & 0.69 \\
\hline Two-h plasma glucose (mM) & $8.83 \pm 1.32$ & $9.02 \pm 1.36$ & 0.34 & $6.57 \pm 1.28$ & $6.35 \pm 1.26$ & 0.41 \\
\hline
\end{tabular}

the dominant and recessive models were significantly associated with an increased risk of susceptibility to GDM $(\mathrm{OR}=2.05,95 \% \mathrm{CI}: 1.20-3.52, p=0.012 ; \mathrm{OR}=1.93,95 \%$ CI: $1.37-2.15, p=0.001 ; \mathrm{OR}=2.62,95 \% \mathrm{CI}: 1.58-4.33$, $p<0.001 ; \mathrm{OR}=1.83,95 \%$ CI: $1.30-2.04, p=0.002)$. Persons with the $\mathrm{C}$ allele have a higher risk of susceptibility to GDM than those with the $\mathrm{T}$ allele $(\mathrm{OR}=2.94,95 \% \mathrm{CI}: 1.87-4.63$, $p<0.001)$. Persons with TNF- $\alpha$ rs1800629 GA and AA genotypes have a higher risk of susceptibility to GDM than those with GG genotypes $(\mathrm{OR}=1.96,95 \% \mathrm{CI}: 1.08-3.57$, $p=0.038 ; \quad \mathrm{OR}=2.05, \quad 95 \% \quad \mathrm{CI}: \quad 1.56-2.17, \quad p<0.001$; $\mathrm{OR}=1.55,95 \% \mathrm{CI}: 1.26-1.82, p<0.001 ; \mathrm{OR}=1.98,95 \% \mathrm{CI}$ : $1.50-2.08, p<0.001)$, and persons with the A allele have a higher risk of susceptibility to GDM than those with the $\mathrm{G}$ allele $(\mathrm{OR}=3.73,95 \% \mathrm{CI}: 2.25-6.18, p<0.001)$.

\section{IL-6 rs1800796, IL-10 rs1800896, and TNF- $\alpha$ rs1800629 interactions}

The MDR analysis of $I L-6$ rs1800796, IL-10 rs1800896, and $T N F-\alpha$ rs 1800629 was designed to measure their interactions with respect to GDM susceptibility. The results of the accuracy $(61.36 \%)$ and sensitivity $(44.21 \%)$ of GDM predicted by the interactions among IL-6 rs1800796, IL-10 rs 1800896 , and TNF- $\alpha$ rs 1800629 are the highest and the specificity $(78.51 \%)$ and precision $(67.30 \%)$ are the lowest. $(\mathrm{OR}=2.91,95 \% \mathrm{CI}: 1.95-4.31, p<0.001)$ (Table 3). In this study, subjects with IL-6 rs1800796 CC, IL-10 rs1800896 TC, and TNF- $\alpha$ rs1800629 GG had the highest risk of GDM susceptibility $(\mathrm{OR}=2.14,95 \% \mathrm{CI}: 1.85-2.65, p=0.005)$. The subjects with IL-6 rs 1800796 CC, IL-10 rs1800896 TT, and TNF- $\alpha$ rs 1800629 GG had the lowest risk of GDM susceptibility (OR $=0.43,95 \%$ CI: $0.35-0.67, p=0.012)$ (Fig. 1A). The cyclic chart analysis results showed that the effects of TNF- $\alpha$ rs1800629, IL-10 rs1800896, and IL-6 rs1800796 on susceptibility to GDM were sequentially reduced (Fig. 1B) and that there is a strong interaction between IL-6 rs 1800796 and TNF- $\alpha$ rs1800629, followed by IL-10 rs1800896 (Fig. 1C).

\section{Correlations between IL-6 rs1800796, IL-10 rs1800896, and TNF- $\alpha$ rs1800629 alleles/genotypes and the clinical characteristics of subjects}

We found that there were no differences in age, gestational weeks, BMI before pregnancy, BMI at birth, newborn body mass, plasma APGAR score, and glucose between GDM patients and the control group with different genotypes at IL-6 rs1800796, IL-10 rs1800896, and TNF- $\alpha$ rs1800629 loci ( $p>0.05$, Tables 4-6).

\section{Plasma inflammatory factor levels}

We used ELISAs to detect the plasma levels of IL-6, IL-10, and TNF- $\alpha$ in all subjects. The results showed that IL-6 plasma levels in GDM patients were significantly higher than those in the control group $(p<0.001$, Fig. 1A) and the area under the curve (AUC) of the working curve

Table 6. Comparison of Clinical Characteristics of the TNF- $\alpha$ Rs1800629 Locus with Different Genotypes

\begin{tabular}{|c|c|c|c|c|c|c|}
\hline & \multicolumn{2}{|c|}{$G D M$} & \multirow[b]{2}{*}{$\mathrm{p}$} & \multicolumn{2}{|c|}{ Control } & \multirow[b]{2}{*}{$\mathrm{p}$} \\
\hline & $G G(\mathrm{n}=191)$ & $G A / A A(\mathrm{n}=51)$ & & $G G(\mathrm{n}=222)$ & $G A / A A(\mathrm{n}=20)$ & \\
\hline Age (years) & $29.29 \pm 4.95$ & $30.31 \pm 5.48$ & 0.20 & $29.64 \pm 4.98$ & $30.75 \pm 3.77$ & 0.09 \\
\hline Gestational weeks & $38.28 \pm 1.46$ & $38.18 \pm 1.56$ & 0.67 & $38.18 \pm 0.80$ & $37.95 \pm 1.00$ & 0.13 \\
\hline BMI before pregnancy $\left(\mathrm{kg} / \mathrm{m}^{2}\right)$ & $23.86 \pm 2.08$ & $23.86 \pm 1.99$ & 1.00 & $23.88 \pm 2.06$ & $23.97 \pm 1.94$ & 0.78 \\
\hline BMI at birth $\left(\mathrm{kg} / \mathrm{m}^{2}\right)$ & $29.80 \pm 2.20$ & $30.44 \pm 2.28$ & 0.07 & $29.66 \pm 3.03$ & $29.72 \pm 3.65$ & 0.90 \\
\hline Newborn body mass (g) & $3302.27 \pm 550.07$ & $3391.65 \pm 552.98$ & 0.30 & $3283.04 \pm 429.81$ & $3402.90 \pm 460.34$ & 0.08 \\
\hline APGAR & $9.80 \pm 0.52$ & $9.78 \pm 0.58$ & 0.81 & $9.78 \pm 0.44$ & $9.80 \pm 0.41$ & 0.77 \\
\hline $\mathrm{HbA1c}(\%)$ & $5.52 \pm 1.11$ & $5.63 \pm 1.20$ & 0.54 & $4.27 \pm 0.97$ & $4.50 \pm 0.89$ & 0.13 \\
\hline Zero-h plasma glucose (mM) & $4.83 \pm 0.69$ & $4.94 \pm 0.70$ & 0.31 & $4.29 \pm 1.21$ & $4.60 \pm 0.99$ & 0.09 \\
\hline One-h plasma glucose $(\mathrm{mM})$ & $10.10 \pm 1.76$ & $10.37 \pm 2.02$ & 0.35 & $7.27 \pm 1.57$ & $7.57 \pm 1.25$ & 0.21 \\
\hline Two-h plasma glucose (mM) & $8.85 \pm 1.35$ & $8.96 \pm 1.26$ & 0.60 & $6.58 \pm 1.28$ & $6.20 \pm 1.20$ & 0.06 \\
\hline
\end{tabular}


(ROC) of the subjects with a diagnosis of GDM for plasma IL-6 levels was 0.8869 (95\% CI: 0.8586-0.9152, $p<0.001$, Fig. 2B). Plasma IL-10 levels in GDM patients were significantly lower than those in the control group $(p<0.001$, Fig. 1C). The AUC of ROC for GDM diagnosis was 0.8052 (95\% CI: $0.7670-0.8433, p<0.001$, Fig. 2D). The plasma TNF- $\alpha$ level in GDM patients was higher than that in the control group $(p<0.001$, Fig. 1E). The AUC of ROC for
GDM diagnosis was 0.8908 (95\% CI: 0.8618-0.9198, $p<0.001$, Fig. 2F).

IL-6 rs 1800796, IL-10 rs1800896, and TNF- $\alpha$ rs 1800629 and plasma IL-6, IL-10, and TNF- $\alpha$ levels

We further analyzed the correlation between plasma inflammatory factor levels and SNP allelic and genotypic
A

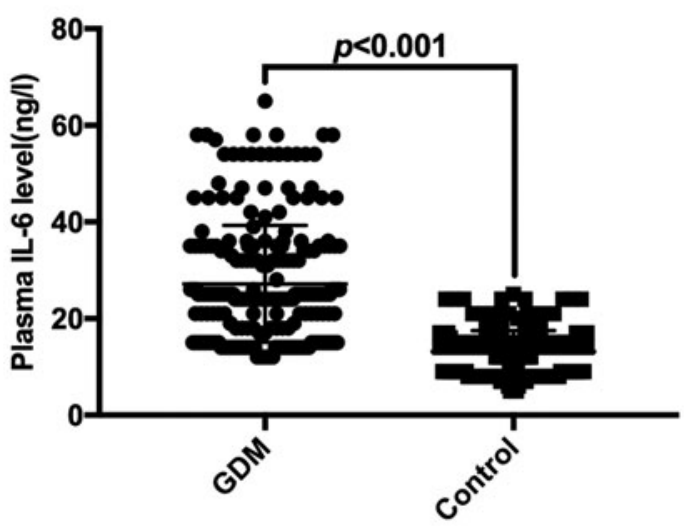

C

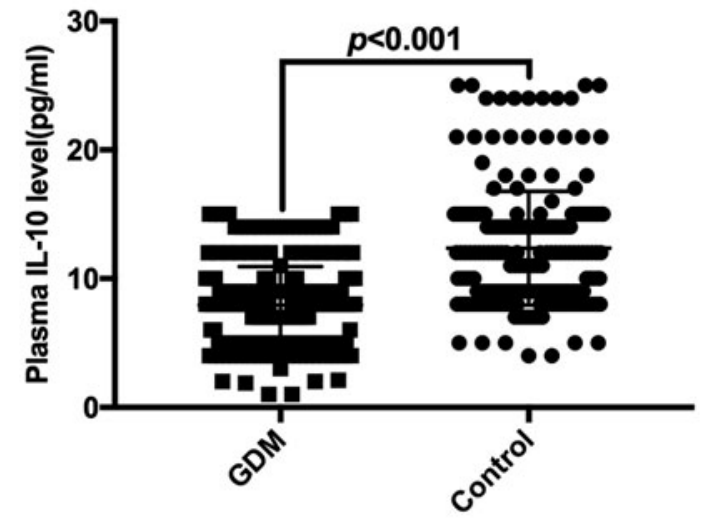

E

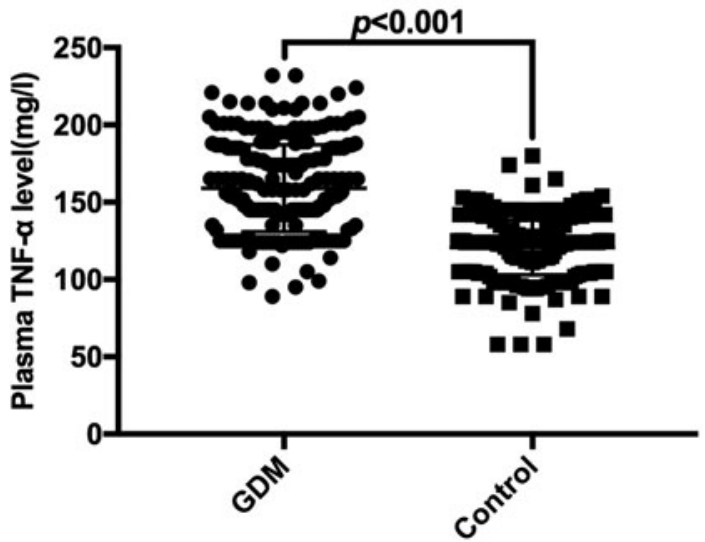

B

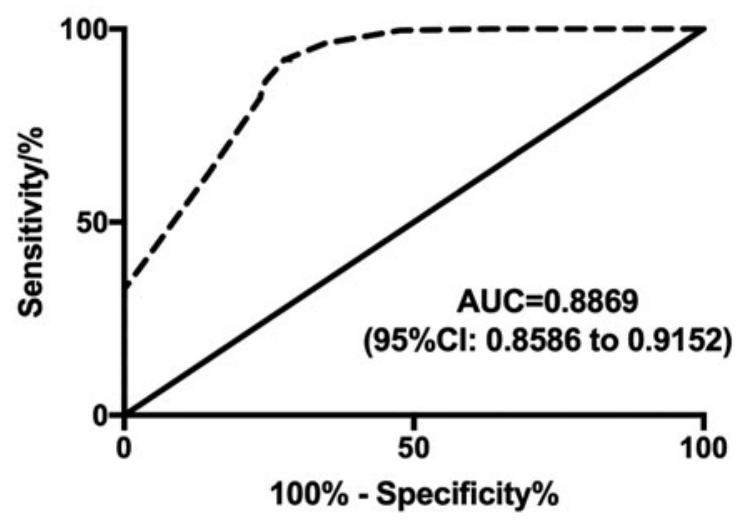

D ROC curve: ROC of Plasma IL-10

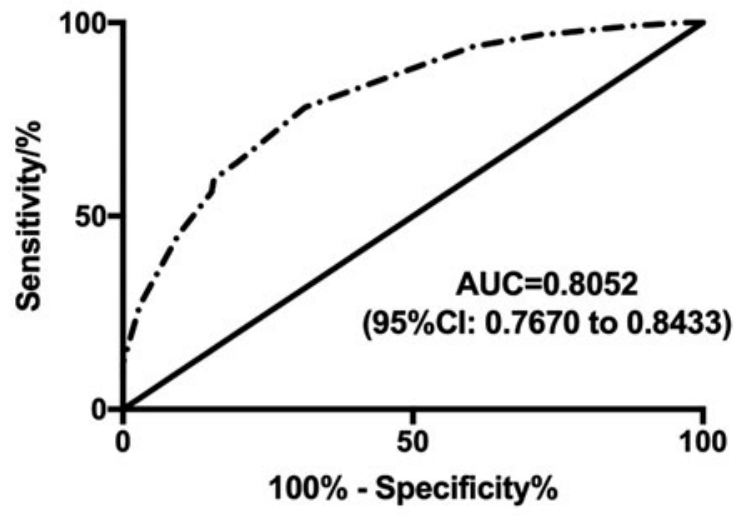

F ROC curve: ROC of Plasma TNF- $\alpha$

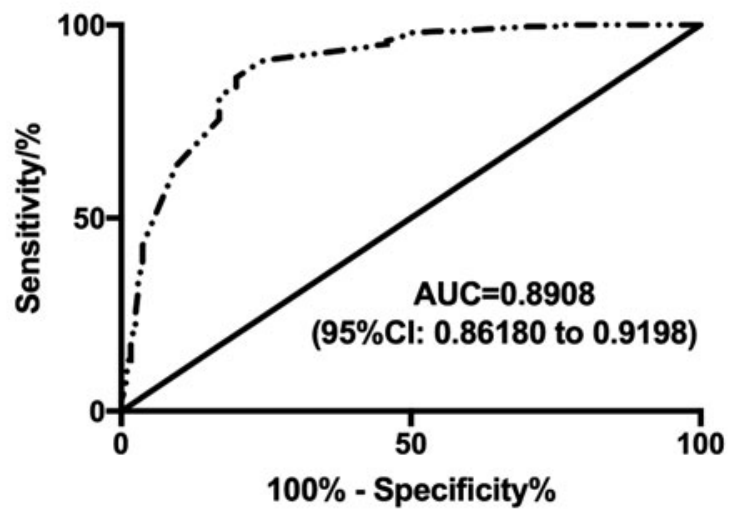

FIG. 2. ELISA detects the levels of IL-6, IL-10, and TNF- $\alpha$ in plasma of subjects. (A) Comparison of IL-6 plasma levels between GDM patients and the control group. (B) ROC of IL-6 plasma levels for a diagnosis of GDM. (C) Comparison of IL-10 plasma levels between GDM patients and the controls. (D) ROC of IL-10 plasma levels for a diagnosis of GDM. (E) Comparison of TNF- $\alpha$ plasma levels between GDM patients and the control group. (F) ROC of TNF- $\alpha$ plasma levels for a diagnosis of GDM. ELISA, enzyme-linked immunosorbent assay; ROC, receiver operating curve. 
FIG. 3. Correlation between IL-6 rs1800796, IL-10 rs1800896, and TNF- $\alpha$ rs1800629 and IL-6, IL-10, and TNF- $\alpha$ plasma levels.

(A) Comparison of IL-6 plasma levels among GDM patients with different genotypes of the IL-6 rs 1800796 locus. (B) Comparison of IL-6 plasma levels among subjects with different genotypes of the IL-6 rs 1800796 locus in the control group.

(C) Comparison of IL-10 plasma levels among GDM patients with different genotypes of the IL-10 rs1800896 locus. (D) Comparison of IL10 plasma levels among subjects with different genotypes of the IL-10 rs1800896 locus in the control group. (E) Comparison of TNF- $\alpha$ plasma levels among GDM patients with different genotypes of the TNF- $\alpha$

rs1800629 locus.

(F) Comparison of TNF- $\alpha$ plasma levels among control subjects with different genotypes at the rs1800629 locus.
A

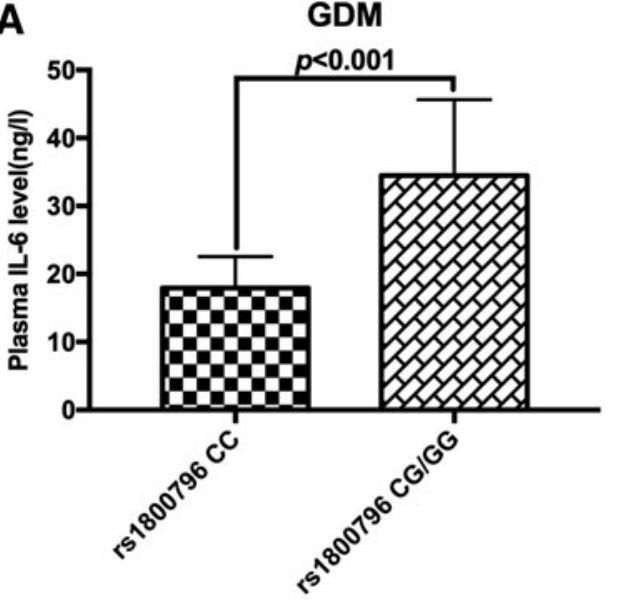

C

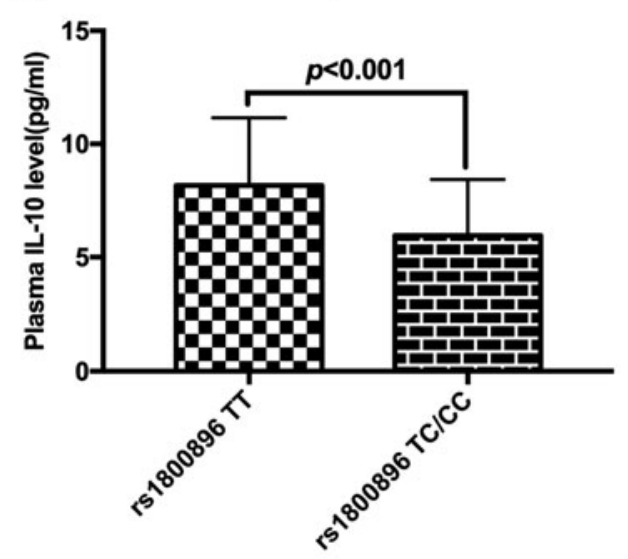

E

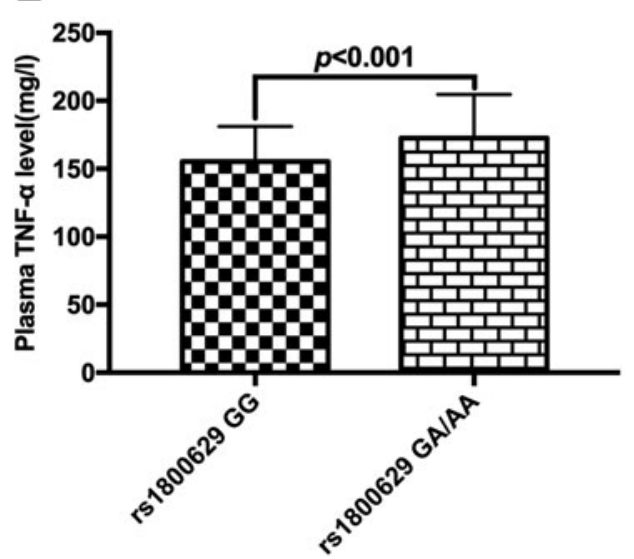

B

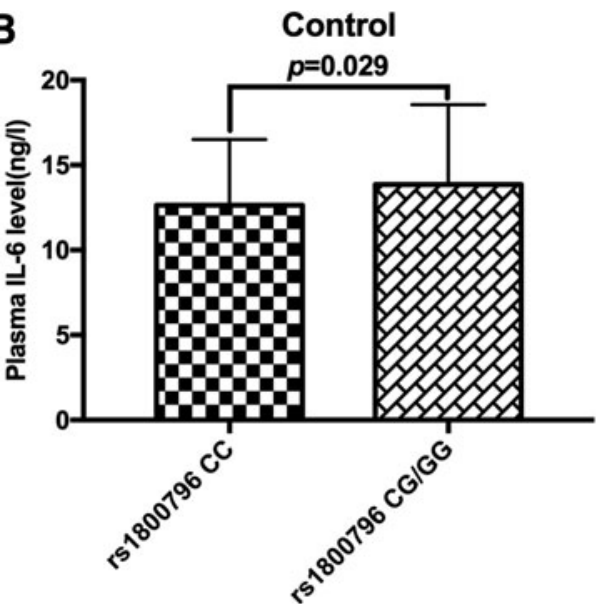

D

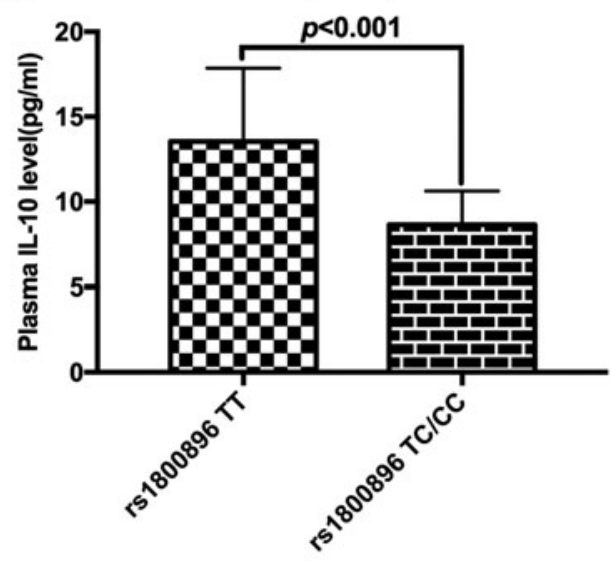

$\mathbf{F}$

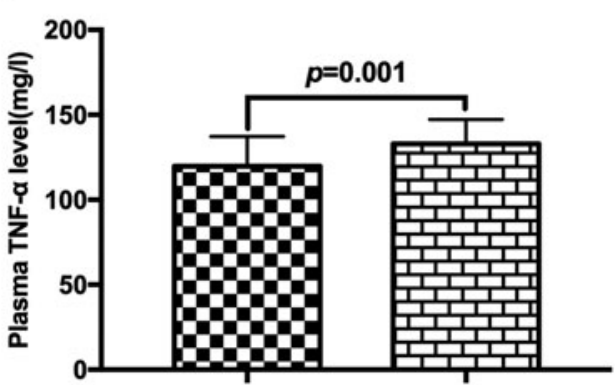

frequencies in the subjects; these results showed that those with the IL-6 rs 1800796 CG/GG genotypes had higher IL-6 plasma levels than those with the CC genotype $(p<0.001$, $p=0.029$; Fig. 3A, B). Persons with the IL-10 rs1800896 TT genotype had higher IL-10 levels than those with the TC/CC genotypes $(p<0.001, p<0.001$; Fig. 3C, D). Persons with the TNF- $\alpha$ rs 1800629 GA/AA genotypes had higher TNF- $\alpha$ levels than those with the GG genotype $(p<0.001, p=0.001$; Fig. 3E, F).

\section{Discussion}

With the exception of trauma, most diseases are the result of a combination of genetic and environmental factors. Different genetic backgrounds can cause different populations to have different susceptibilities to certain diseases. The study of human genetic polymorphisms can help us understand the distribution of the different genotypes of various genes in different diseases. 
In this study of the Han Chinese, we found that subjects with the IL-6 rs1800796 G allele had higher plasma IL-6 levels and a higher risk of GDM susceptibility than subjects with the $\mathrm{C}$ allele. Persons with the IL-10 rs1800896 C allele had lower IL-10 plasma levels than persons with the T allele and a higher risk of GDM susceptibility. Subjects with the TNF- $\alpha$ rs1800629 A allele had higher plasma TNF- $\alpha$ levels and a higher risk of susceptibility to GDM than subjects with the $\mathrm{G}$ allele.

IL-6 is an inflammatory cytokine with multiple biological functions, with a molecular weight of $26 \mathrm{kD}$. Mature IL-6 contains 184 amino acids, and the amino acid residues at positions $1-28$ have no known important functions. The main role of the 97-104-amino acid region is to maintain the structural integrity of the protein. The 29-34-amino acid residue region is in close proximity to the 180-184-amino acid residue, which constitutes the IL- 6 active site and plays a key role in binding to the receptor through which it exerts its biological functions (Hammacher et al., 1994). The results of this study show that subjects with the IL-6 rs $1800796 \mathrm{G}$ allele had a higher risk of susceptibility to GDM than those who carry the $\mathrm{C}$ allele, and ELISA test results showed that $I L-6$ rs1800796 G allele carriers had higher plasma IL-6 levels, which are consistent with results of the study by Ding $e t a l$. (2019). The reason for this finding is likely that the -572 locus of the $I L-6$ promoter region affects the transcriptional efficiency of $I L-6$, and the G-allele at this site corresponds to a higher level of promoter activity because its cognate transcription factors bind with more affinity, which promotes increased transcription. IL-6 may cause GDM by promoting insulin resistance (Bastard et al., 2002). In addition, it has been found that during development of type 2 diabetes, IL- 6 can stimulate liver cells to produce acute response proteins and activate the innate immune system, thereby promoting a positive feedback cycle that further enhances the circulating levels of IL-6 and other inflammatory proteins in the body (Lindsay et al., 2001), including the C-reactive protein. These two proteins can interact to affect the body's immune system and produce diabetes-related conditions such as GDM (Kesika et al., 2019; Kianpour et al., 2019).

In this study, we found that subjects with the IL-10 rs $1800896 \mathrm{C}$ allele had lower IL-10 plasma levels and higher GDM susceptibility than T allele subjects. Zhu et al. (2019) showed that the IL-10 rs1800896 C allele is associated with higher IL-10 levels in plasma and peripheral blood mononuclear cell culture supernatants, which is the exact opposite of the results of this study. The specific reason is unclear and may be related to the expression of IL-10 by other factors.

We found that carriers of the TNF- $\alpha$ rs1800629 A allele had higher TNF- $\alpha$ plasma levels and greater risk of GDM than carriers with the $\mathrm{G}$ allele. We believe that the reason may be that the A allele is more efficient in binding to transcription factors and the resulting increased expression of TNF- $\alpha$ is higher. TNF- $\alpha$ can destroy islet B cells in autoimmunity, which is a defect of B cell secretion function (Kuhl, 1998). TNF- $\alpha$ can also reduce insulin secretion by reducing insulin receptor autophosphorylation and promoting phosphorylation of the receptor matrix protein serine. Another possibility is that in islet $\beta$-cells, TNF- $\alpha$ can reduce glucose-stimulated insulin secretion (Laukkanen et al., 2004). Although islet B cells secrete insulin normally because TNF- $\alpha$ can increase insulin resistance (Laukkanen et al., 2004), to maintain stable blood glucose levels, islet B cells secrete more insulin to compensate for lower blood glucose. Persistent hyperglycemia gradually inhibits islet $\beta$-cell secretion, which eventually leads to impaired islet $\beta$-cell function and to a gradual decline in insulin secretion. The amount of insulin cannot counterbalance insulin resistance, leading to an increase in blood glucose.

In addition, in this study, it was also found that the interactions among the IL- 6 rs 1800796 , IL-10 rs1800896, and TNF- $\alpha$ rs1800629 loci affect susceptibility to GDM, indicating that GDM is a polygenic trait.

This study is not without its limitations. First, the sample size is relatively small, thus additional studies with larger sample sizes should be conducted. Second, there is a lack of evidence from in vitro and in vivo studies supporting the correlation of IL-6 rs1800796, IL-10 rs1800896, and TNF- $\alpha$ rs1800629 with their respective protein expression levels.

\section{Conclusion}

In this study, we found that the IL-6 rs $1800796 \mathrm{G}$, IL-10 rs $1800896 \mathrm{C}$, and TNF- $\alpha$ rs1800629 A alleles were significantly associated with an increased risk of susceptibility to GDM.

\section{Author Disclosure Statement}

No competing financial interests exist.

\section{Funding Information}

This work was supported by a grant from the Traditional Chinese Medical Science and Technology Plan of Zhejiang Province (2016ZQ018).

\section{References}

Bastard JP, Maachi M, Van Nhieu JT, et al. (2002) Adipose tissue IL-6 content correlates with resistance to insulin activation of glucose uptake both in vivo and in vitro. J Clin Endocrinol Metab 87:2084-2089.

Chang L, Lan T, Wu L, et al. (2015) The association between three IL-6 polymorphisms and HBV-related liver diseases: a meta-analysis. Int J Clin Exp Med 8:17036-17045.

Charles BA, Doumatey A, Huang H, et al. (2011) The roles of IL-6, IL-10, and IL-1RA in obesity and insulin resistance in African-Americans. J Clin Endocrinol Metab 96:E2018E2022.

Ding Y, Feng Q, Chen J, Song J (2019) TLR4/NF-kappaB signaling pathway gene single nucleotide polymorphisms alter gene expression levels and affect ARDS occurrence and prognosis outcomes. Medicine (Baltimore) 98:e16029.

Feng Y, Feng Q, Qu H, et al. (2020) Stress adaptation is associated with insulin resistance in women with gestational diabetes mellitus. Nutr Diabetes 10:4.

Hammacher A, Ward LD, Weinstock J, et al. (1994) Structurefunction analysis of human IL-6: identification of two distinct regions that are important for receptor binding. Protein Sci 3: 2280-2293.

International Association of Diabetes and Pregnancy Study Groups Consensus Panel. (2010) International association of diabetes and pregnancy study groups recommendations on the diagnosis and classification of hyperglycemia in pregnancy. Diabetes Care 33:676-682. 
Jin X, Yin S, Zhang Y (2019) Association between the IL-101082G/A, IL-10-819T/C and IL-10-592A/C polymorphisms and Brucellosis susceptibility: a meta-analysis. Epidemiol Infect 147:e316.

Kalani M, Choopanizadeh M, Rasouli M (2019) Influence of genetic variants of gamma interferon, interleukins 10 and 12 on Visceral Leishmaniasis in an endemic area, Iran. Pathog Glob Health 113:14-19.

Kesika P, Sivamaruthi BS, Chaiyasut C (2019) Do probiotics improve the health status of individuals with diabetes mellitus? A review on outcomes of clinical trials. Biomed Res Int 2019:1531567.

Kianpour M, Saadatmand F, Nematbakhsh M, Fahami F (2019) Relationship between C-reactive protein and screening test results of gestational diabetes in pregnant women referred to health centers in Isfahan in 2013-2014. Iran J Nurs Midwifery Res 24:360-364.

Kowalski GM, Nicholls HT, Risis S, et al. (2011) Deficiency of haematopoietic-cell-derived IL-10 does not exacerbate highfat-diet-induced inflammation or insulin resistance in mice. Diabetologia 54:888-899.

Kuhl C (1998) Etiology and pathogenesis of gestational diabetes. Diabetes Care 21 Suppl 2:B19-B26.

Lacroix M, Lizotte F, Hivert MF, et al. (2019) Calcifediol decreases interleukin- 6 secretion by cultured human trophoblasts from GDM pregnancies. J Endocr Soc 3:2165-2178.

Laffer B, Bauer D, Wasmuth S, et al. (2019) Loss of IL-10 promotes differentiation of microglia to a M1 phenotype. Front Cell Neurosci 13:430.

Laukkanen O, Pihlajamaki J, Lindstrom J, et al. (2004) Common polymorphisms in the genes regulating the early insulin signalling pathway: effects on weight change and the conversion from impaired glucose tolerance to Type 2 diabetes. The Finnish Diabetes Prevention Study. Diabetologia 47: 871-877.

Lindsay RS, Krakoff J, Hanson RL, et al. (2001) Gamma globulin levels predict type 2 diabetes in the Pima Indian population. Diabetes 50:1598-1603.

Ma Y, Tang RK, Yang X, et al. (2011) Lack of an association between interleukin-6 gene promoter polymorphisms ($174 \mathrm{G} / \mathrm{C},-572 \mathrm{G} / \mathrm{C})$ and ischemic heart disease and/or ischemic stroke: a meta-analysis. Hum Immunol 72:641-651.

McIntyre HD, Catalano P, Zhang C, et al. (2019) Gestational diabetes mellitus. Nat Rev Dis Primers 5:47.

Nergiz S, Altinkaya OS, Kucuk M, et al. (2014) Circulating galanin and IL-6 concentrations in gestational diabetes mellitus. Gynecol Endocrinol 30:236-240.

Rancourt RC, Ott R, Ziska T, et al. (2020) Visceral adipose tissue inflammatory factors (TNF-alpha, SOCS3) in gestational diabetes (GDM): epigenetics as a clue in GDM pathophysiology. Int J Mol Sci 21:479.
Revilla M, Obach V, Cervera A, et al. (2002) A-174G/C polymorphism of the interleukin-6 gene in patients with lacunar infarction. Neurosci Lett 324:29-32.

Sacks DB (2014) Diagnosis of gestational diabetes mellitus: it is time for international consensus. Clin Chem 60:141-143.

Shahriyari E, Vahedi L, Roshanipour N, et al. (2019) Exploring the association of IL-10 polymorphisms in Behcet's disease: a systematic review and meta-analysis. J Inflamm (Lond) 16: 26.

Tang L, Xu S, Li P, Li L (2019) Predictors of insulin treatment during pregnancy and abnormal postpartum glucose metabolism in patients with gestational diabetes mellitus. Diabetes Metab Syndr Obes 12:2655-2665.

Wang Z, Li Y, Zhou Y, Qiao Y (2019) Association between the IL-10 rs1800872 polymorphisms and periodontitis susceptibility: a meta-analysis. Medicine (Baltimore) 98:e17113.

Warzocha K, Ribeiro P, Bienvenu J, et al. (1998) Genetic polymorphisms in the tumor necrosis factor locus influence non-Hodgkin's lymphoma outcome. Blood 91:3574-3581.

Yang SJ, Kim TN, Baik SH, et al. (2013) Insulin secretion and insulin resistance in Korean women with gestational diabetes mellitus and impaired glucose tolerance. Korean J Intern Med 28:306-313.

Yang Y, Liu L, Liu B, et al. (2018) Functional defects of regulatory $\mathrm{T}$ cell through interleukin 10 mediated mechanism in the induction of gestational diabetes mellitus. DNA Cell Biol $37: 278-285$.

Zhang H, Kuchroo V (2019) Epigenetic and transcriptional mechanisms for the regulation of IL-10. Semin Immunol 44: 101324

Zhang M, Zhou Y, Zhong J, et al. (2019) Current guidelines on the management of gestational diabetes mellitus: a content analysis and appraisal. BMC Pregnancy Childbirth 19:200.

Zhu SW, Liu ZJ, Sun QH, Duan LP (2019) Effect of the interleukin 10 polymorphisms on interleukin 10 production and visceral hypersensitivity in Chinese patients with diarrheapredominant irritable bowel syndrome. Chin Med J (Engl) 132:1524-1532.

Address correspondence to: Heng Chen, MD Department of Clinical Laboratory The Second Affiliated Hospital of Zhejiang Chinese Medical University 318 Chaowang Road Hangzhou 310005 Zhejiang China

E-mail: cnchss@163.com 